\title{
Construction of Single-Phase Nickel Disulfide Microflowers as High-Performance Electrodes for Hybrid Supercapacitors
}

\author{
Ziyang Dai,${ }^{\dagger}$ Lichun Xue,${ }^{\dagger}$ Zhenbao Zhang, ${ }^{\dagger}$ Yang Gao, ${ }^{\dagger}$ Jian Wang,${ }^{\S}$ \\ Qingsheng Gao, ${ }^{*, \dagger}$ and Dengjie Chen ${ }^{*, \dagger}$
}

${ }^{\dagger}$ College of Chemistry and Materials Science, Jinan University, Guangzhou 510632,

China

College of Materials Science and Engineering, Hunan University, Changsha 410082,

China

${ }^{\S}$ Department of Chemistry, College of Science, Seoul National University, Seoul 08826, South Korea

*Corresponding authors.

*Email: tqsgao@jnu.edu.cn.

*Email: dengjie.chen@jnu.edu.cn. 


\section{Materials and Methods}

\subsection{Synthesis of the Precursor for Nickel-Based Microflowers.}

The precursor for the synthesis of nickel-based microflowers was prepared through a hydrothermal method. Typically, $12.0 \mathrm{mmol}$ of $\mathrm{Ni}\left(\mathrm{NO}_{3}\right)_{2} \cdot 6 \mathrm{H}_{2} \mathrm{O}, 12.0 \mathrm{mmol}$ of $\mathrm{NH}_{4} \mathrm{~F}$ and $30.0 \mathrm{mmol}$ of urea were dissolved in $80.0 \mathrm{~mL}$ of deionized (DI) water under stirring to form a green transparent solution, which was then transferred into a Teflon-lined stainless steel autoclave and heated at $110{ }^{\circ} \mathrm{C}$ for $8 \mathrm{~h}$ in an oven. After the reaction, the precipitate was centrifuged, washed several times with DI water and ethanol, and finally dried in an oven at $60{ }^{\circ} \mathrm{C}$ for $10 \mathrm{~h}$ to obtain the green precursor powder.

\subsection{Synthesis of NiS Microflowers.}

First, $480.36 \mathrm{mg}$ of $\mathrm{Na}_{2} \mathrm{~S} \cdot 9 \mathrm{H}_{2} \mathrm{O}$ was dissolved in $35.0 \mathrm{~mL}$ of DI water, followed by adding $92.71 \mathrm{mg}$ of the obtained green precursor into the solution. The mixture was transferred into a Teflon-lined stainless steel autoclave and heated at $160{ }^{\circ} \mathrm{C}$ for $10 \mathrm{~h}$ in an oven. After the reaction, the precipitate was separated by centrifugation, washed several times with DI water and ethanol, and placed in an oven at $60{ }^{\circ} \mathrm{C}$ for $10 \mathrm{~h}$ to yield the dried black powder.

\subsection{Formation of $\mathrm{NiS}_{2}$ Microflowers.}

Typically, $927.1 \mathrm{mg}$ of thioacetamide (TAA) was dissolved in $35.0 \mathrm{~mL}$ of DI water, followed by a homogeneous distribution of $92.71 \mathrm{mg}$ of the green precursor. The mixture was transferred into a Teflon-lined stainless steel autoclave and heated at $180{ }^{\circ} \mathrm{C}$ for $10 \mathrm{~h}$ in an oven. After the reaction, the precipitate was separated by 
centrifugation, washed several times with DI water and ethanol, and placed in an oven at $60{ }^{\circ} \mathrm{C}$ for $10 \mathrm{~h}$ to obtain the dried black powder.

\section{Materials Characterizations.}

X-ray diffraction (XRD) patterns were acquired on an X-ray powder diffractometer (Rigaku Smartlab, $3 \mathrm{~kW}$ ), using $\mathrm{Cu} \mathrm{K} \alpha$ radiation at $40 \mathrm{kV}$ and $40 \mathrm{~mA}$. Field emission scanning electron microscope (FE-SEM, ULTRA 55, ZEISS) and high-resolution transmission electron microscope (HR-TEM, JEM-2010, JEOL) were employed to investigate the morphology and structure. Nitrogen adsorption-desorption isotherms were measured on an Autosorb-iQ at $77 \mathrm{~K}$. X-ray photoelectron spectroscopy (XPS, PHI5000 VersaProbe) was obtained to analyze the oxidation state of surface chemical species. The $\mathrm{C} 1 \mathrm{~s}$ peak at $284.8 \mathrm{eV}$ was used as the reference for all spectra.

\section{Computational Details.}

Density functional theory (DFT) calculations were carried out within the spin-polarized generalized gradient approximation (GGA) of Perdew, Burke, and Ernzerhof (PBE) as implemented in the Vienna Ab-initio Simulation Package (VASP). ${ }^{1}$ Core electrons were handled using the projector augmented wave (PAW) method with an energy cutoff of $450 \mathrm{eV}^{2}$ The localized Ni $3 \mathrm{~d}$ electronic interaction was treated with DFT $+\mathrm{U}$ method with $\mathrm{U}_{\text {eff }}=6.2 \mathrm{eV} \cdot{ }^{3}$ Gaussian smearing was used with a width of $0.05 \mathrm{eV}$ to determine the partial occupancies. To calculate the density of states, the geometries were first relaxed until the energy difference was smaller than $10^{-6} \mathrm{eV}$ and forces smaller than $0.01 \mathrm{eV}^{-1}$, followed by the static calculations 
with the relaxed structure. The obtained CHG and CHGCAR files were used for the non-self-consistent calculation to obtain the density of states. The Brillouin zone was sampled with $15 \times 15 \times 11,7 \times 7 \times 19$, and $11 \times 11 \times 11$ Monkhorst-Pack $k$-point meshes for $\alpha-\mathrm{NiS}, \beta-\mathrm{NiS}$, and $\mathrm{NiS}_{2}$, respectively.

\section{Electrochemical Measurements.}

To fabricate the electrode, the active material, carbon black, and polyvinylidene fluoride (PVDF) with mass ratio of 8: 1: 1 were mixed in $100 \mu \mathrm{L}$ of $\mathrm{N}$-methyl pyrrolidone, which was ground to form the slurry. We used the brush to paste the slurry onto a nickel foam current collector and placed it in an oven at $60{ }^{\circ} \mathrm{C}$ for $10 \mathrm{~h}$ to form a working electrode. The mass loading of the active material on the nickel

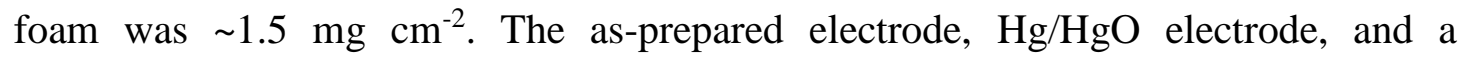
platinum wire were used as the working electrode, reference electrode, and counter electrode, respectively. The electrochemical measurements were carried out in a three-electrode system using $2 \mathrm{M} \mathrm{LiOH}$ as the electrolyte. Cyclic voltammetry (CV), galvanostatic charge-discharge (GCD) and electrochemical impedance spectroscopy (EIS, 1-100,000 Hz) measurements were carried out on a CHI 660E electrochemical workstation, and the cycling stability was evaluated on a LANHE CT2001A instrument. The calculation of specific capacity is based on the equation of $Q_{s}=I t / m$, where $\mathrm{Q}_{\mathrm{s}}, \mathrm{I}$, $\mathrm{t}$, and $\mathrm{m}$ are the specific capacity, the discharge current, the discharge time, and the mass of the active material, respectively.

\section{Assembly and Evaluation of HSCs.}

The $\mathrm{NiS}_{2} / / \mathrm{AC}$ HSCs were fabricated by assembling the $\mathrm{NiS}_{2}$ positive electrode 
and AC negative electrode in a two-electrode system, with $2 \mathrm{M} \mathrm{LiOH}$ as the electrolyte. Using the relation of $\mathrm{Q}^{+}=\mathrm{Q}^{-}$to determine the mass ratio of the positive electrode material to the negative electrode material, where $\mathrm{Q}^{+}$refers to the capacity of the positive electrode and $\mathrm{Q}^{-}$refers to the capacity of the negative electrode. The specific capacitance, energy density and power density of HSCs were obtained based on the equations of $\mathrm{C}_{\mathrm{s}}=\mathrm{It} / \mathrm{mV}, \mathrm{E}_{\mathrm{s}}=1 / 2 \mathrm{C}_{\mathrm{s}} \mathrm{V}^{2}$, and $\mathrm{P}_{\mathrm{s}}=\mathrm{E}_{\mathrm{s}} / \mathrm{t}$, respectively, where $\mathrm{C}_{\mathrm{s}}$ represents the specific capacitance, I refers to the discharge current, $\mathrm{t}$ is the discharge time, $\mathrm{m}$ is the sum of the mass of the positive and negative active materials, $\mathrm{V}$ is the voltage window, $\mathrm{E}_{\mathrm{s}}$ is the energy density, and $\mathrm{P}_{\mathrm{s}}$ is the power density. 
Table S1. Rietveld refinement results of the as-prepared NiS and $\mathrm{NiS}_{2}$ microflowers.

\begin{tabular}{|c|c|c|c|}
\hline Sulfides & \multicolumn{2}{|c|}{$\mathrm{NiS}$} & $\mathrm{NiS}_{2}$ \\
\hline $\mathrm{R}_{\mathrm{p}}(\%)$ & \multicolumn{2}{|c|}{3.01} & 3.10 \\
\hline $\mathrm{R}_{\mathrm{wp}}(\%)$ & \multicolumn{2}{|c|}{3.79} & 3.89 \\
\hline$\chi^{2}$ & \multicolumn{2}{|c|}{1.06} & 1.08 \\
\hline Phases & $\alpha$ & $\beta$ & pyrite \\
\hline $\mathrm{a}(\AA)$ & 3.41 & 9.62 & 5.68 \\
\hline $\mathrm{b}(\AA)$ & 3.41 & 9.62 & 5.68 \\
\hline$c(\AA)$ & 5.26 & 3.15 & 5.68 \\
\hline Fract. (\%) & 14.65 & 85.35 & 100 \\
\hline
\end{tabular}

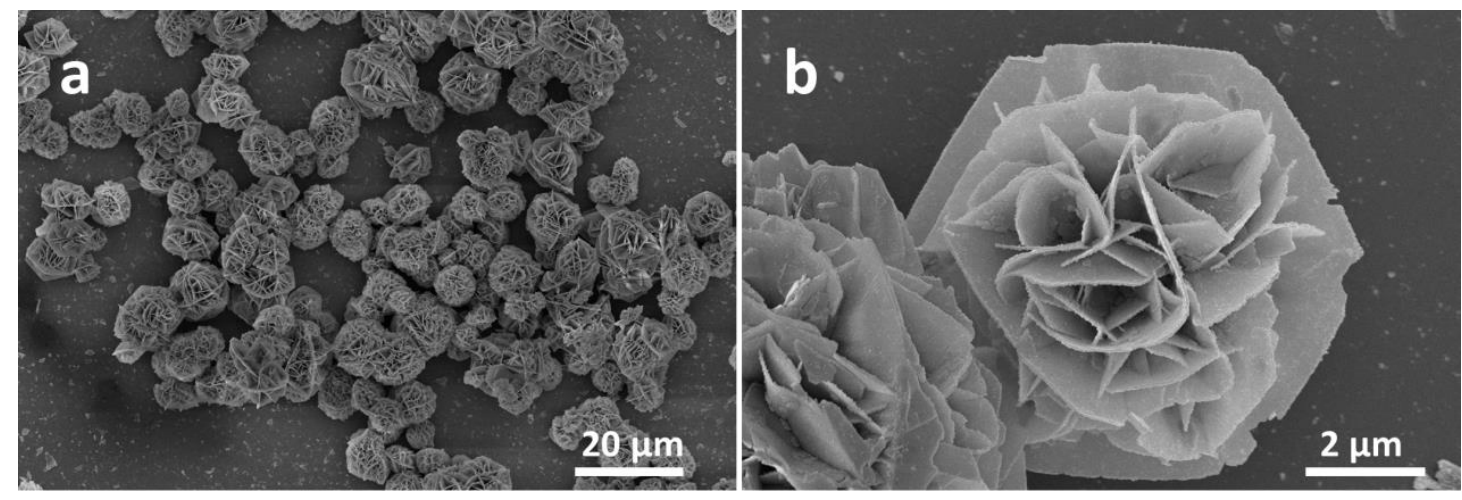

Figure S1. FE-SEM images of the precursor with a unique structure of microflowers. 

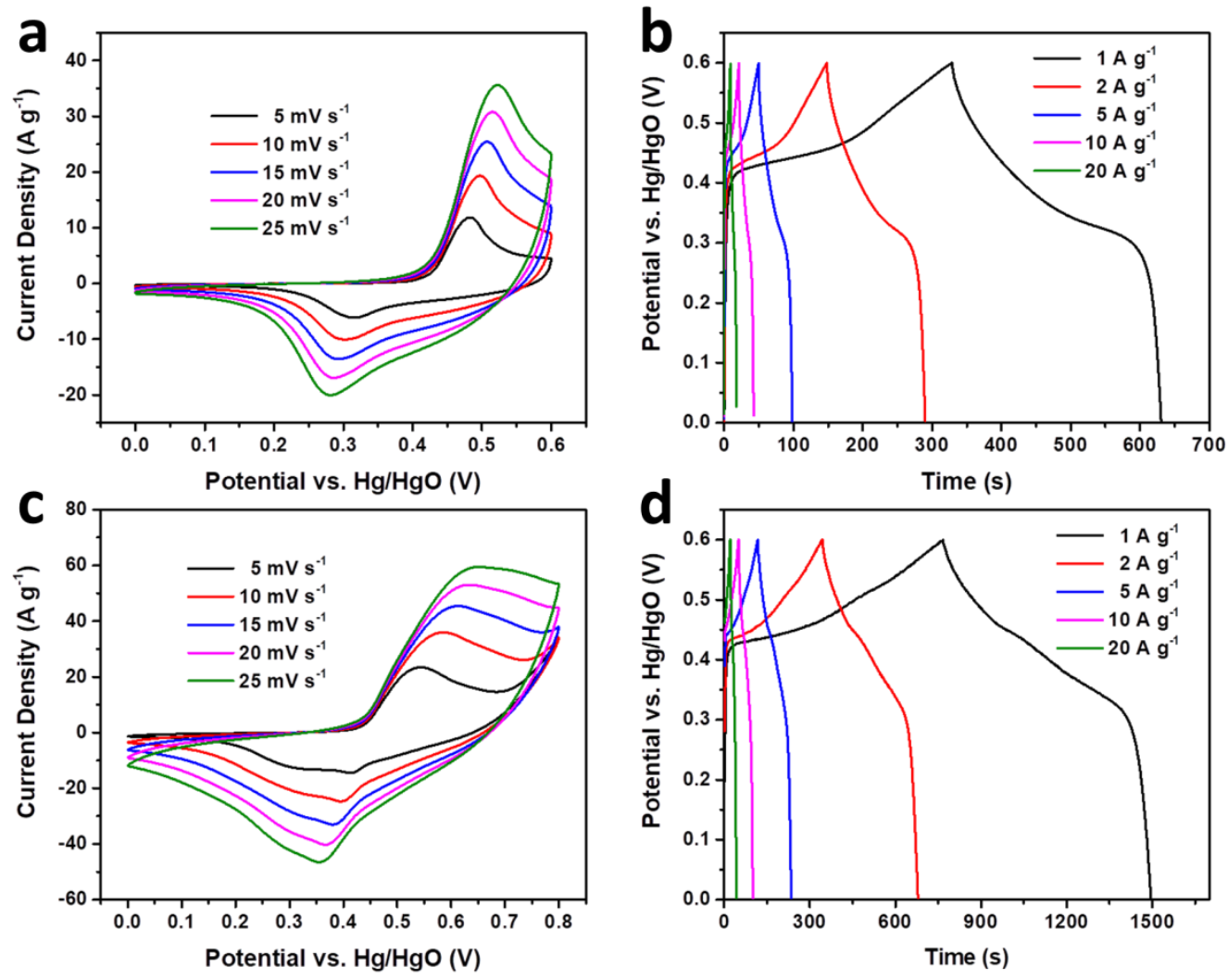

Figure S2. CV curves of (a) the precursor and (c) the NiS microflowers, and GCD curves of (b) the precursor and (d) the NiS microflowers.

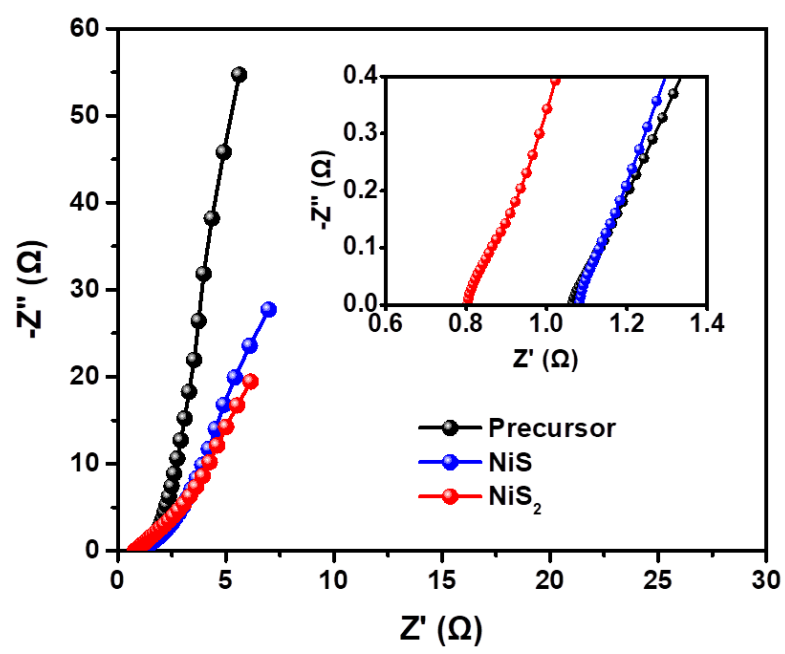

Figure S3. Nyquist plots of the precursor, $\mathrm{NiS}$ and $\mathrm{NiS}_{2}$ microflowers electrodes. 

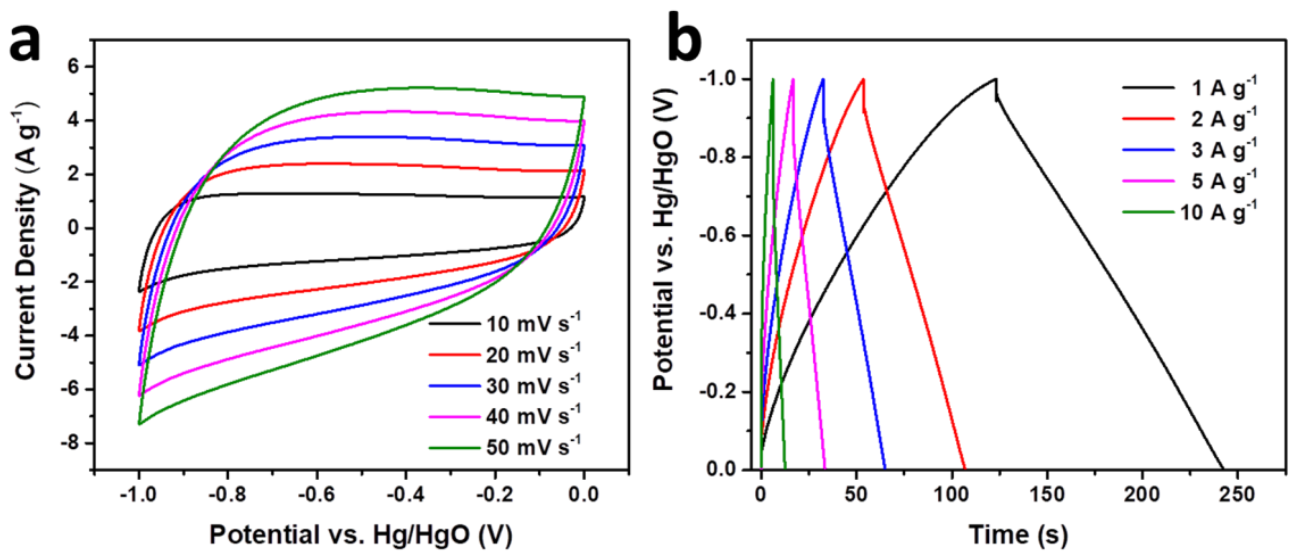

Figure S4. (a) CV and (b) GCD curves of the AC.

\section{References}

(1) Perdew, J. P.; Burke, K.; Ernzerhof, M., Generalized gradient approximation made simple. Phys. Rev. Lett. 1996, 77, 3865.

(2) Blöchl, P. E., Projector augmented-wave method. Phys. Rev. B 1994, 50, 17953.

(3) Wang, M.; Navrotsky, A., Enthalpy of formation of $\mathrm{LiNiO}_{2}, \mathrm{LiCoO}_{2}$ and their solid solution, $\mathrm{LiNi}_{1-\mathrm{x}} \mathrm{Co}_{\mathrm{x}} \mathrm{O}_{2}$. Solid State Ionics 2004, 166, 167-173. 